\title{
Faire revenir à la vie
}

Gestes d'urgence et corps instruments du secours (France, 1957-1965)

Bring back to life. Emergency gestures and rescue instruments (France, 1957-1965)

\section{Charles-Antoine Wanecq}

\section{(2) OpenEdition Journals}

Édition électronique

URL : http://journals.openedition.org/artefact/846

DOI : $10.4000 /$ artefact.846

ISSN : 2606-9245

\section{Éditeur :}

Association Artefact. Techniques histoire et sciences humaines, Presses universitaires du Midi

\section{Édition imprimée}

Pagination : 115-132

ISBN : 978-2-7535-7305-5

ISSN : 2273-0753

Référence électronique

Charles-Antoine Wanecq, «Faire revenir à la vie », Artefact [En ligne], 6 | 2017, mis en ligne le 31 mai 2018, consulté le 04 mars 2020. URL : http://journals.openedition.org/artefact/846 ; DOI : https:// doi.org/10.4000/artefact.846 


\section{Faire revenir à la vie. Gestes d'urgence et corps instruments du secours (France, 1957-1965)}

Charles-Antoine WANECQ*

\section{Résumé}

Autour de 1960, le bouche-à-bouche et le massage cardiaque externe, dont la pertinence est d'abord mise en évidence aux États-Unis, sont reconnus par le corps médical français comme les deux méthodes les plus efficaces en situation de détresse vitale et adoptés pour standardiser l'enseignement des premiers secours. À partir de l'étude de la légitimation de ces procédés, cet article propose d'explorer le processus de construction scientifique et institutionnelle de la notion d'urgence médicale dans la France de l'après-guerre, à partir des dimensions techniques qui contribuent à sa définition.

Mots-clés : histoire du corps, premiers secours, protection civile, réanimation, sauvetage, technique, urgence médicale.

\section{Abstract. Bring back to life. Emergency gestures and rescue instruments (France, 1957-1965)}

First used and legitimized in the United States, mouth-to-mouth and external cardiac massage were recognized by the French medical body as the two most efficient methods of resuscitation at the turn of the 1960s in case of vital emergency, and selected to standardize first-aid training. From the study of the legitimisation of these techniques, this article intends to explore the process of scientific and institutional elaboration of the notion of medical emergency in post-war France, by examining the technical dimensions which contributed to its definition.

*. Charles-Antoine Wanecq est agrégé d'histoire et doctorant au Centre d'histoire de Sciences Po où il prépare une thèse sur l'histoire de la notion d'urgence médicale en France, de l'entre-deux-guerres aux années 1980, sous la direction de Paul-André Rosental. En 2016-2017, il est ATER à l'université Paris Diderot. 
Keywords : civil defense, first-aid, history of the body, lifesaving, medical emergency, resuscitation technique.

Que faire en présence d'un malade ou blessé inanimé? Face au péril ou à la mort apparente, les stratégies développées par les sociétés pour prendre en charge les victimes révèlent l'état des savoirs physiologiques aussi bien que le contexte technique, politique et social dans lesquels elles se trouvent. Permettant de saisir l'essor de l'organisation des secours au XVIII ${ }^{\mathrm{e}}$ siècle dans les villes européennes, en particulier pour les noyés, une riche historiographie a mis en évidence le rôle décisif des sauveteurs bénévoles, organisés dans des sociétés de bienfaisance, qui apparaissent comme des techniciens du retour à la vie, sur un terrain où les médecins ne s'aventurent guère ${ }^{1}$. Au sein des sociétés de sauvetage qui se multiplient au cours du XIXe siècle se transmettent des savoirs et des techniques encore largement usités dans la France des années 1950. Ce n'est qu'à partir de l'entre-deux-guerres que les sapeurs-pompiers prennent une part active dans la lutte contre les asphyxiés, à cause des nouveaux facteurs de risque d'asphyxie qu'entraîne la diffusion de l'électricité et du gaz dans les habitations et sur les lieux de travail'2. La revue de la littérature médicale anglo-saxonne et française permet d'identifier une abondante bibliographie sur l'histoire de la respiration artificielle et des méthodes de réanimation cardio-pulmonaire, qui se focalise souvent sur les médecins ayant contribué aux expérimentations et à la fixation des gestes de premiers secours ${ }^{3}$.

Cependant, la question du retour à la vie des individus en détresse vitale, asphyxiés ou en arrêt cardiaque, dépasse largement le cercle de la sphère médicale, dans la mesure où n'importe qui peut devenir témoin d'un accident et, par là même, responsable et acteur de l'aide à la victime. L'étude du rapport entre corps sains et corps blessés permet alors d'interroger les évolutions des limites savantes et sociales de la mort ${ }^{4}$. Les travaux scientifiques mesurant le temps entre l'accident et la prise en charge par les sauveteurs démontrent que l'efficacité des secours est en grande partie liée à leur rapidité : si $95 \%$ des asphyxiés sont réanimés lors d'une prise en charge dans la minute de l'intervention, et $50 \%$ dans les quatre premières minutes, la victime secourue au-delà de six minutes après son accident n'a plus que $1 \%$ de chance de survivre (et aucune chance au-delà de huit minutes ${ }^{5}$ ). Dès lors, la formation des citoyens aux gestes de premiers secours devient un enjeu essentiel de protection des populations, afin de rendre capable le plus grand nombre d'intervenir dès le moment de l'accident auprès de personnes atteintes d'une défaillance cardiaque ou respiratoire nécessitant une assistance rapide. Au-delà, il convient de rappeler que, dans un contexte sanitaire de meilleure maîtrise des maladies épidémiques et chroniques, la part des accidents s'accroît considérablement parmi les causes de mortalité ${ }^{6}$. La lutte contre les " morts violentes ${ }^{7}$ » constitue alors un des axes de la politique de santé publique, à la suite des expériences de guerre dans lesquelles les civils ont été la cible des attaques ${ }^{8}$. Rompus à l'inter- 
vention dans des situations d'urgence', les médecins militaires ou formés en période de conflit jouent dans cette histoire un rôle important.

Cet article a pour ambition de proposer une analyse historienne de la définition de nouvelles normes en matière de premiers secours, en se focalisant sur la séquence 1957-1965 qui constitue un moment matriciel dans l'édification d'une doctrine de la réanimation cardiopulmonaire, à partir du cas de deux gestes spécifiques, le bouche-à-bouche et le massage cardiaque externe. Ils se trouvent légitimés de manière quasisimultanée aux États-Unis et en Europe par des médecins qui leur confèrent une valeur supérieure aux méthodes alors en vigueur. Nous interrogerons donc le rôle spécifique du monde médical dans l'adoption de ces techniques par les associations de secourisme au début des années 1960 : dans un contexte de stabilisation de leurs savoirs et d'institutionnalisation de leur discipline, les réanimateurs s'engagent dans des débats circonscrits jusqu'alors au monde des sauveteurs ${ }^{10}$. Il s'agit dès lors de mettre en lumière les dynamiques par lesquelles le bouche-à-bouche et le massage cardiaque externe ont été reconnus en France comme les méthodes les plus efficaces pour sauver un maximum d'individus par une partie du corps médical comme par différentes institutions en charge de la sécurité et de la protection des populations (service de santé de l'armée, ministère de la Santé, Académie de médecine...), aux moyens disparates et aux visions souvent conflictuelles. Par ailleurs, la création d'un brevet national de moniteur de secourisme en $1964^{11}$, censé standardiser les savoirs secouristes à l'échelle du territoire, consacre la légitimité de ces deux gestes, en les sélectionnant parmi tout un ensemble d'autres méthodes qu'il devient superflu d'enseigner. Ainsi, nous tenterons de réfléchir au processus de fabrication d'un consensus autour de ces techniques de premiers secours, à partir des notions discutées en sociologie de la santé d'efficacité ${ }^{12}$ et de standardisation ${ }^{13}$ de ces gestes.

Cette recherche se situe donc au croisement de l'histoire des techniques et des savoirs, de l'histoire sociale de la médecine et de l'histoire du corps ${ }^{14}$. Elle se fonde sur une lecture des archives des départements ministériels et d'associations de secourisme (Croix-Rouge française, publications de la Fédération nationale de sauvetage et de l'Ordre de Malte), croisée avec les productions scientifiques, émanant de lieux d'échange et de légitimation, comme l'Académie de médecine, les colloques et la presse médicale ${ }^{15}$. 


\section{Face à la mort : du secourisme aux principes de la réanimation}

\section{Retour sur les héritages des pratiques de respiration artificielle à la fin des années 1950}

Si le tournant des années 1960 constitue un moment essentiel de la stabilisation d'une doctrine des premiers secours, il convient de revenir sur les pratiques en vigueur dans la décennie précédente, plurielles et variables selon les traditions secouristes. Les méthodes utilisées datent pour beaucoup de la fin du XIX $x^{e}$ siècle, même si certaines améliorations techniques sont intervenues dans l'entredeux-guerres. Le secourisme a connu un essor considérable lors de la Seconde Guerre mondiale avec la mise en place des équipes d'urgence de la Croix-Rouge française, née en 1940 de l'unification de trois anciennes sociétés de secours aux blessés ${ }^{16}$. Après la guerre, alors qu'elle est intervenue pour porter secours les victimes de nombreux bombardements, l'association s'impose dans le champ en formant une grande partie des secouristes, suivie de près par la Fédération nationale de sauvetage. Àla fin des années 1950, elles délivrent chacune un peu plus de 30000 diplômes de secourisme par an, assurant une sorte d'hégémonie dans le domaine ${ }^{17}$, à côté d'un ensemble d'associations de taille variable. Prenant la suite de la Défense passive en 1951, le Service national de la protection civile, dont l'histoire reste en grande partie à écrire, tente de se faire une place dans le domaine en créant sa propre certification, notamment pour les pompiers qui dépendent directe- ment de lui. Bien que peu dotée en crédits au cours de la période comparativement à d'autres pays ${ }^{18}$, cette démarche se situe dans un contexte de Guerre froide et de guerres de décolonisation : ces dernières impriment leur marque sur les institutions et les individus impliqués dans la gestion des populations et de leurs vulnérabilités.

Pour autant, chacune de ces structures dispose de ses propres formations et de ses manuels, mettant à l'honneur plusieurs méthodes de respiration artificielle dont l'enseignement repose sur le choix des médecins et moniteurs. En France, c'est le risque d'accident électrique qui a fait entrer les méthodes de réanimation dans la règlementation française, à la suite des réunions d'une commission créée par les ministères des Travaux publics et de $\mathrm{l}^{\prime}$ Industrie pour fixer les instructions à donner aux agents en cas d'électrocution. Mise en concurrence avec la méthode de Silvester, la méthode de Schafer se trouve officiellement préconisée en France par le décret du 23 janvier 1927, dans les cas d'électrocution ${ }^{19}$. Elle consiste pour le sauveteur à se placer à califourchon sur les hanches de la victime étendue sur le ventre, bras en avant, tête sur le côté, et à exercer une quinzaine de fois par minute de fortes pressions au niveau des reins. Il est recommandé aux sauveteurs de poursuivre ce geste aussi longtemps que possible, pendant plusieurs heures parfois, jusqu'à ce qu'un médecin déclare la mort de la victime en cas de non réanimation. Elle a pour principal avantage d'être facilement 
praticable par le plus grand nombre $a$ priori, sans risque de complication, et d'être moins fatigante que la méthode de Silvester. Pratiquée depuis la fin du $\mathrm{XIX}^{\mathrm{e}}$ siècle sur des individus couchés sur le dos, cette dernière nécessite des sauveteurs qu'ils réalisent des tractions énergiques et répétées des bras, ce qui est physiquement éprouvant. La minimisation de l'énergie dépensée apparaît ainsi comme un argument essentiel de l'adoption d'un procédé au détriment $\mathrm{d}^{\prime}$ un autre ${ }^{20}$. Le médecin-commandant Charles Cot, médecin du régiment des sapeurs-pompiers de Paris, a introduit en France, en 1923, la méthode de Schafer, en la faisant tester par les hommes du rang puis adopter dans le décret de 1927 comme procédé à enseigner et appliquer partout en France, copiant en cela les pays anglo-saxons. En fait, Cot privilégie cette méthode pour des raisons pédagogiques: constatant que les asphyxies au gaz sont de plus en plus nombreuses dans les appartements parisiens ${ }^{21}$, il doit enseigner en peu de temps un procédé simple à reproduire par les pompiers et gardiens de la paix appelés au secours des victimes ${ }^{22}$. Par ailleurs, la méthode de Laborde, qui consiste en des tractions rythmées de la langue, est fréquemment utilisée, en complément des deux autres. La fixation du nom et du contenu précis de chacune d'elles fait l'objet de débats et de controverses parmi les autorités médicales et au sein des congrès internationaux de sauvetage, notamment celui qui se tient à Copenhague en 1934. Àcette occasion, en effet, le colonel Holger Nielsen présente la méthode qu'il a mise au point pour améliorer celle de Schafer (elle consiste en une compression thoracique avec mouvement des bras) et qui tend à largement s'imposer dans l'enseignement dans les années 1950 en France. Si les autorités valident tel ou tel procédé en raison des résultats d'études comparées, il n'en demeure pas moins que, sur le terrain, les sauveteurs doivent avant tout recourir aux techniques qui leur sont plus familières, pour gagner en efficacité.

Alors que quinze mille personnes meurent chaque année en France de syncope asphyxique dans les années $1950^{23}$, la recherche de la meilleure méthode de réanimation doit dépendre tant des caractéristiques physiques et des aptitudes des sauveteurs que du contexte de l'accident. En 1956, Pierre Marion et Raymond Deleuze suggèrent à leurs collègues anesthésistes d'encourager les sauveteurs isolés à recourir au procédé de Silvester, indiquant déjà son rôle dans la stimulation circulatoire : " par de simples compressions dans l'aire cardiaque, il est possible de réaliser de véritables massages indirects du cœur ${ }^{24}$. " Remise en cause par les découvertes médicales du début des années $1950^{25}$, qui pointent précisément la supériorité d'un procédé qui agit également sur la circulation sanguine, la méthode de Schafer demeure officiellement recommandée jusqu'en 1978, en dépit du vœu adopté par l'Académie nationale de médecine dès 1954 pour son abrogation ${ }^{26}$. Le décret de 1927 est alors remplacé par un arrêté qui remet à l'honneur la méthode de Silvester, néanmoins revue, tout en recommandant surtout l'usage du bouche-à-bouche. Entre temps, néanmoins, la création du brevet de moniteur de secourisme en 1964 s'est imposée comme un autre vecteur de standardisation des techniques. 


\section{Des appareils au secours des limites des sauveteurs}

Si les méthodes de respiration artificielle peuvent s'effectuer à mains nues, elles nécessitent souvent une certaine endurance de la part des sauveteurs qui doivent attendre l'autorisation du médecin pour faire cesser les soins. Pour pallier cet inconvénient, plusieurs machines, conçues par des médecins et des ingénieurs, sont employées par les sauveteurs et dans les corps de sapeurspompiers chargés de porter secours aux asphyxiés ${ }^{27}$. À Paris, les hôpitaux font ainsi intervenir auprès de leurs patients les hommes de la brigade avec les appareils d'oxygénation qu'ils utilisent sur les lieux mêmes des accidents : jusque dans les années 1950, l'Assistance publique est ainsi dépendante des techniques des militaires pour dispenser ce type de soins à ses malades ${ }^{28}$.

Trois appareils, inventés en France dans l'entre-deux-guerres, connaissent un certain succès et sont diffusés dans les centres de secours ${ }^{29}$. Le premier, reconnu par l'Académie de médecine en 1923, est celui du docteur Panis, ancien interne des hôpitaux de Paris, qui permet d'appliquer la méthode de Schafer sur des asphyxiés, en supprimant "l'incompétence et la fatigue ${ }^{30}$ » des sauveteurs. Il s'agit d'un châssis métallique activé par des leviers, sur lequel la victime est placée en position ventrale. Il provoque une ample ventilation pulmonaire, par compression et décompression du thorax, maintenu dans des sangles. Fort de son expérience auprès des hommes du régiment, le docteur Cot met au point en 1931 un appareil très semblable qui connaît un certain succès dans les corps de sapeurs-pompiers et les équipes de secouristes qui se le procurent (fig. 1). Enfin, le pulmoventilateur de Charles

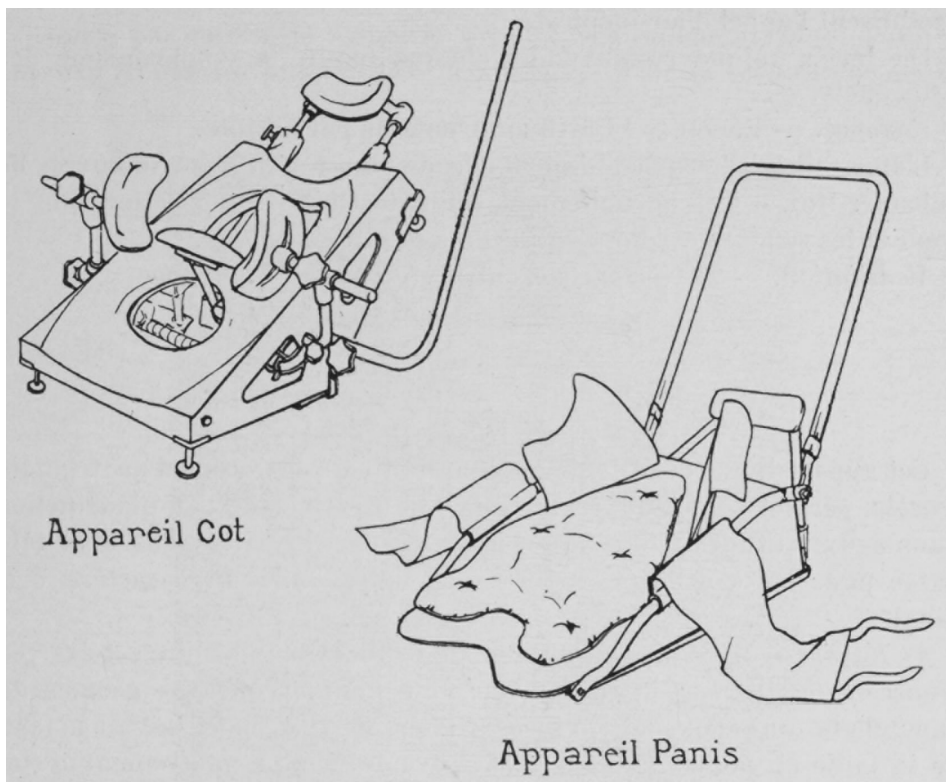

Figure 1. - Appareils de Cot et de Panis. Médecin en chef HEDERER, « La respiration artificielle. Étude critique et expérimentale », Bulletin de l'Académie de médecine, vol. 98, n 23 p. 861. 
Hederer, médecin-général de la Marine, tente de combiner les méthodes de Schafer et de Silvester, en introduisant l'élévation des épaules de la victime ${ }^{31}$. L'indication " à plat ventre » ou " poitrine ", apposée en gros caractères sur la partie centrale de la plateforme, ne doit en principe laisser aucun doute au secouriste (fig. 2). Permettant aux sauveteurs d'accomplir plus facilement leur mission, ces machines constituent un prolongement de leur geste et de leur corps et un remède aux limites du " moteur humain ${ }^{32} »$. Ces machines - et le dispositif potentiellement effrayant qu'elles constituent $^{33}$ - correspondent à une séquence particulière au cours de laquelle certains médecins ont tenté de mécaniser les méthodes manuelles de respiration artificielle. À partir de 1957, ces appareils ne sont plus mentionnés parmi les méthodes employées en cas d'asphyxie dans les statistiques nationales de la Protection Civile, signe de leur progressive désaffection.
Alors que ces techniques se fondent sur l'expiration forcée, d'autres procédés de réanimation des asphyxiés reposent sur l'oxygénation. Depuis le $\mathrm{xvIII}^{\mathrm{e}}$ siècle, plusieurs méthodes, utilisant notamment des soufflets, consistent à insuffler de l'air dans l'organisme des malades dont le système respiratoire est défaillant. À la suite d'une série d'expériences, le directeur du laboratoire de physiologie appliquée de Yale, Yandell Henderson, propose dans les années 1920 d'associer $5 \%$ de gaz carbonique à l'oxygène pour réanimer les asphyxiés. Le mélange obtenu, appelé " carbogène ", permettrait de stimuler la ventilation : il est ainsi utilisé dès 1931 par les pompiers de Paris. On peut replacer les expérimentations des années 1930 dans le contexte de préparation active de la lutte contre les effets d'une potentielle guerre chimique ${ }^{34}$. La création des services de Défense passive contribue fortement aux entreprises de recherche, d'une part, et, d'autre part, d'investis-

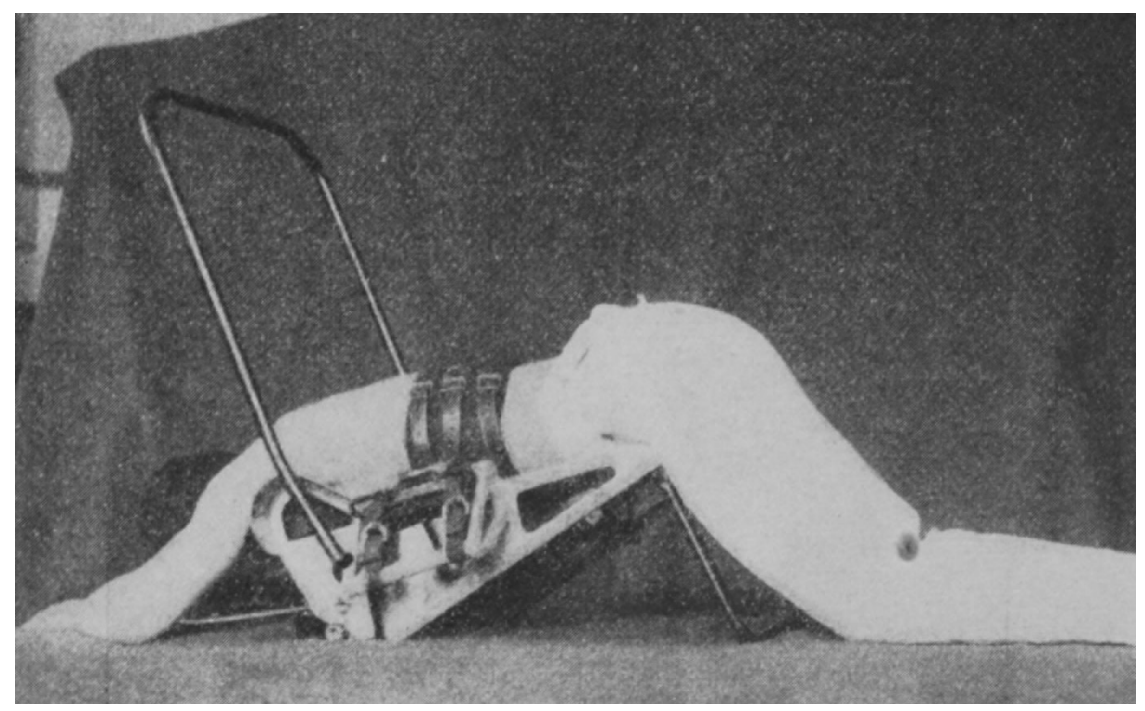

Figure 2. - Le pulmoventilateur de Charles Hederer. Médecin en chef Hederer, art. cité, p. 864. 
sements en formation et en matériel dans les corps locaux de sapeurs-pompiers. Le médecin-général Genaud, à la tête du service médical de la Protection civile dans les années 1950, a d'ailleurs commencé sa carrière à l'organisation sanitaire de la Défense passive dans les années 1930 aux côtés de Cot. Dans un contexte de préparation de moyens de défense contre la guerre atomique ${ }^{35}$, il poursuit ses recherches et demeure en dialogue constant avec le bureau de l'enseignement de la Protection civile, chargé de l'élaboration des programmes du brevet de secourisme.

$\mathrm{Si}$ la Seconde Guerre mondiale a permis des avancées en réanimation ${ }^{36}$, notamment par la rencontre et les échanges entre médecins anglo-américains et français, l'épidémie de poliomyélite qui se répand depuis Copenhague à partir de 1952 constitue le cadre des plus notables avancées dans la discipline : certains malades risquant l'asphyxie par la paralysie des muscles respiratoires contraignent les soignants à rester à leur chevet avec un ballon pour assurer leur ventilation. Ceci rend nécessaire la mise au point de machines pouvant suppléer l'homme, mais nécessitant la présence d'un médecin réanimateur. La taille des appareils constitue un obstacle à leur déplacement sur les lieux où se trouve le malade : ainsi, lors de l'épidémie de poliomyélite, le ventilateur mis au point par le médecin suédois Engström ne peut être déplacé ${ }^{37}$. Quelques ambulances sont adaptées pour recevoir un poumon d'acier, vaste structure dans laquelle certains malades se trouvaient enfermés parfois pendant plusieurs années ${ }^{38}$. Il devient nécessaire de concevoir des appareils plus petits et plus maniables : l'ingénieur parisien Pesty fabrique alors son Respirateur à pause réglable, ou Rosentiel-Pesty-Richard, connu sous le nom de R. P. R., simple à l'emploi et léger (il ne pèse que douze kilogrammes ${ }^{39}$ ). Il est utilisé dès mai 1956 par les médecins de l'hôpital Necker qui, sous la direction du professeur Cara, sont chargés d'assurer en urgence le transport des poliomyélitiques ${ }^{40}$. Depuis le laboratoire expérimental de physique de l'Assistance publique qu'il dirige, le professeur Cara assure l'entretien des appareils de ventilation et contribue à la diffusion du R. P. R. dans les services spécialisés des hôpitaux français ${ }^{41}$. La machine devient un support des fonctions vitales du malade dans les services de réanimation, les unités de soins intensifs qui se développent alors en France, mais aussi à l'intérieur des ambulances des hôpitaux et des Services mobiles d'urgence et de réanimation (SMUR) qui se mettent en place dans les années qui suivent. Pour autant, l'essor de ces instruments renouvelle le rôle pré-médical décisif des secouristes, censés agir dans les instants qui suivent l'accident avant de passer le relais aux médecins lourdement équipés. 


\section{La rupture de 1960 : deux innovations en réanimation cardio-respiratoire}

\section{La redécouverte d'un geste efficace, le bouche-à-bouche}

Reposant sur le principe de l'insufflation d'air expiré, le bouche-à-bouche a l'avantage de pouvoir être pratiqué très facilement par n'importe quel sauveteur, sans recours à du matériel. Le renversement de la tête doit permettre la libération des voies respiratoires pour éviter que la langue ne fasse obstruction au passage de l'air en tombant au fond de la gorge. En référence au livre des Rois, nombreux sont les articles qui, retraçant l'histoire de cette technique, pointent ses origines bibliques ${ }^{42}$. Peut-être est-ce une manière de convaincre les lecteurs et potentiels usagers du caractère ancestral et authentique de cette méthode tombée en désuétude. Plusieurs communications à l'Académie de Médecine au cours du XIX ${ }^{e}$ siècle attestent cependant de sa fréquente utilisation sur des nouveau-nés dont la fragilité rend dangereuse toute manipulation énergique. Nécessitant un contact délicat du sauveteur et de la personne à sauver, le bouche-à-bouche présente aussi l'avantage de pouvoir être pratiqué sur des individus à immobiliser en raison de probables fractures. Les expériences menées aux États-Unis autour du docteur Safar ${ }^{43}$, considéré comme le père de la réanimation cardio-respiratoire ${ }^{44}$, permettent de réévaluer l'efficacité de la méthode et assurent sa promotion dans de nombreuses publications et communications à partir de $1958^{45}$.

Anesthésiste formée dans un environnement très masculin auprès de méde- cins américains lors de la Libération de la France, le docteur Yvonne Noviant effectue des voyages annuels aux ÉtatsUnis, en Angleterre et en Scandinavie, où la discipline est nettement plus avancée qu'en France ${ }^{46}$. Par ses contributions dans des colloques et des revues médicales, elle sensibilise ses confrères français, d'abord sceptiques, à cette méthode frappée de discrédit ${ }^{47}$. Rapidement, début 1959, le docteur Charles François, médecin chef du service médical d'Électricité de France (EDF), se fait également le promoteur de cette technique, lors d'une réunion tenue au ministère de la Santé à la suite du décès d'un agent d'EDF, où s'affrontent les visions de plusieurs médecins dont Genaud, de la Protection civile ${ }^{48}$. Le bouche-à-bouche est ensuite présenté à la Faculté de médecine de Paris,le 9 juin 1960, par le docteur Elam ${ }^{49}$, en présence du doyen. À l'Académie de médecine, un mois plus tard, la séance du 5 juillet 1960 est pour une grande partie consacrée à l'étude comparée des différents procédés : six d'entre eux sont présentés par le médecin-colonel Lartigue. Nourri de la lecture des travaux de Safar et Elam dont il reprend les conclusions, son propos se fonde également sur les expérimentations d'enseignement pratique et généralisé de respiration artificielle qu'il a réalisées depuis cinq ans avec les hommes de l'École militaire d'Antibes et du bataillon de Joinville. Sous l'égide de ses médecins, le monde militaire apparaît ici comme un laboratoire des expérimentations en réanimation de victimes, 
mais la présence dans ce tableau d'une jeune femme issue du milieu hospitalier, comme celle d'un médecin d'EDF, manifeste la diversité des acteurs impliqués et de leurs cultures professionnelles. Cependant, la normalisation d'une technique de respiration artificielle est l'occasion pour les médecins intéressés par ces questions de réévaluer avantages et inconvénients comparés de chacune d'elle, en fonction du débit respiratoire qu'elles permettent, mais surtout, en fait, de la situation dans laquelle se trouvent la victime et le sauveteur.

\section{Le massage cardiaque externe, révélation ou invention?}

Si le bouche-à-bouche est une redécouverte, le massage cardiaque externe est une trouvaille ou au moins la normalisation d'un acte qui n'était pas pratiqué jusqu'alors pour lui-même. La réanimation cardiaque relevait exclusivement $\mathrm{du}$ domaine des spécialistes, cardiologues et médecins capables d'effectuer un massage cardiaque intra-thoracique : il s'agissait pour ces derniers d'inciser le thorax du malade afin de saisir son cœur et de le palper, lors d'opérations chirurgicales en particulier. Les observations réalisées par les médecins permettent de souligner l'importance de la rapidité d'intervention après l'arrêt cardiaque, afin d'oxygéner le sang. Longtemps, la confusion entre asphyxie et arrêt du cœur persiste : ainsi, les compressions du thorax sont réalisées comme moyens de respiration artificielle plutôt que pour favoriser la circulation ${ }^{50}$. Pourtant, les frictions et les gestes appliqués lors de la respiration artificielle permettaient de faire un massage cardiaque « sans le savoir ».
Comme Safar et Elam pour le bouche-à-bouche, l'ingénieur américain Kouwenhoven est le premier à proposer, en 1960, une analyse de la technique du massage cardiaque externe, rendant possible sa normalisation ${ }^{51}$. Il réalise vingt réanimations humaines avec succès, obtenues grâce à des massages à la fréquence de soixante par minute, associés au bouche-à-bouche. L'expérimentation permet ainsi de formuler un discours médical sur le geste, ce qui contribue à lui conférer le statut d'acte thérapeutique. Pour autant, un des enjeux de son utilisation est bien de le rendre praticable par le plus grand nombre, au-delà des seuls médecins : puisqu'aucun matériel n'est nécessaire, il devient possible, comme pour le bouche-à-bouche, d'en faire un acte secouriste à enseigner largement. Ces gestes et leur codification s'inscrivent pour partie dans le processus de spécialisation des disciplines médicales, et en particulier ici l'institutionnalisation de l'anesthésie et de la réanimation ${ }^{52}$. Pourtant, si les réanimateurs jouent ainsi un rôle important dans la transmission de ces nouveaux gestes en France, ils sont loin d'être les seuls puisque, comme on l'a vu, le service de santé des armées, le comité médical d'EDF et les associations de secourisme s'y intéressent également. Le bouche-à-bouche et le massage cardiaque externe font leur entrée parmi les méthodes utilisées par les pompiers dans les statistiques nationales de la Protection civile en 1961, ce qui laisse penser qu'ils ont été rapidement considérés comme des techniques efficaces. Avec ces deux nouveaux gestes, standardisés plutôt qu'inventés, le corps du sauveteur doit s'ger dans le sauvetage et devenir luimême technique ${ }^{53}$. Cette brève séquence, 
autour de 1960, permet donc de réfléchir aux usages et aux formes de la réanimation cardio-respiratoire, tandis que les mains et la bouche demeurent de véritables « instruments ${ }^{54}$ » lors de malaises et autres accidents.

\section{Du débat sur l'efficacité à l'appropriation des techniques}

\section{Gouverner le corps des sauveteurs}

Reconnu comme la méthode la plus efficace, le bouche-à-bouche doit désormais infuser toutes les structures qui se préoccupent de la lutte contre la mort par asphyxie, les associations de sauveteurs comme le corps médical. Ses promoteurs insistent sur l'importance de trouver les moyens de vaincre la répugnance engendrée par le contact de la bouche du sauveteur volontaire avec celle du blessé ou du malade ${ }^{55}$. En effet, beaucoup de responsables des instances qui gèrent le secourisme en France se montrent réticents à l'idée de pratiquer cette technique, à commencer par le médecin-général Genaud qui, dans une note destinée aux responsables du Service national de la protection civile, manifeste des doutes sur le caractère pertinent de l'usage de la méthode pour cette raison:

« C'est un procédé certainement très efficace, mais l'expérience m'a montré qu'il n'était pas possible de l'enseigner, pour des raisons de répugnance évidentes. C'est pourquoi il n'a pas paru indiqué de l'inscrire au programme des brevets de Secouriste. Le dégoût serait sans doute pire s'il s'agissait d'un moribond ou d'un cadavre ${ }^{56}$. »

L'acceptabilité par le public, y compris un public averti de secouristes, est donc posée comme un critère essentiel de diffusion de la méthode ${ }^{57}$. Plusieurs parades sont alors trouvées : le bouche-à-nez, la plus évidente, permet d'éviter le contact du sauveteur avec la bouche du malade qui, dans bien des cas, saigne ou vomit. Certains recommandant l'emploi d'un tissu fin laissant passer l'air, tandis que l'emploi d'une canule laisse une distance entre les deux bouches. Enfin, l'usage d'un équipement constitué d'un masque et d'un ballon, selon l'anesthésiste suédois Arne Ruben, permet d'éviter le contact entre la bouche du sauveteur et celle de la victime ${ }^{58}$. L'objet, y compris le plus banal, devient un rouage essentiel de la technique ${ }^{59}$.

Lors des séances de formation, il est plus commode de se servir d'un mannequin plutôt que de solliciter un des élèves. C'est une innovation scandinave qui est mise à l'honneur : le mannequin Ressusci-Anne est conçu, en 1961, par un fabricant de poupées et de jouets suédois qui a sauvé son jeune fils de la noyade ${ }^{60}$. Une de ses connaissances, le Docteur Lind, a eu l'idée de le solliciter, alors que son collègue Safar venait de publier ses 
articles sur le bouche-à-bouche. La forme même de ce mannequin représente un enjeu important pour les formateurs : elle doit reproduire les dimensions d'un individu moyen, celui que l'on a le plus de chance de secourir. Par ailleurs, d'après les concepteurs de Ressusci-Anne, le choix d'un faciès féminin permettrait de vaincre la répugnance des hommes, plus réticents, semble-t-il, à pratiquer le geste sur un de leurs semblables. Grâce à la formation et à l'entraînement, l'anticipation des gestes à accomplir permet de fluidifier le comportement de celui qui les pratique et d'accroittre son efficacité. Il s'agit, pour le sauveteur, d'apprendre à ajuster son corps en peu de temps à celui de l'individu en détresse ${ }^{61}$. Après l'avoir vu tester lors d'un de ses nombreux voyages en Suède, Yvonne Noviant présente à ses confrères français l'utilité de cet objet, progressivement adopté dans les hôpitaux et les centres de secours. L'apprentissage mobilise la vue et le toucher des sauveteurs qui développent une véritable intelligence pratique à l'occasion des régulières séances d'entraînement ${ }^{62}$.

Face à une Protection civile peu active dans le soutien à l'enseignement de cette nouvelle technique, le ministère de la Santé demande aux préfets de veiller dans leurs départements à la création d'un centre d'enseignement du boucheà-bouche et fournit les mannequins nécessaires aux anesthésistes-réanimateurs chargés d'enseigner les techniques de premiers secours à partir de 1963 , se positionnant ainsi dans le champ du secourisme en conférant une autorité aux médecins spécialistes, affirmant ainsi une forme de médicalisation du secourisme $^{63}$. Dans un contexte de lutte contre les conséquences des accidents de la circulation dont le nombre et la gravité ne cessent de croître $^{64}$, la circulaire diffusée le 11 mars 1963 par le bureau des secours d'urgence du ministère de la Santé continue de préconiser l'enseignement des méthodes de Silvester, de Nielsen, mais insiste surtout sur l'apprentissage du bouche-à-bouche. La création, en 1964, d'une commission de la réanimation au sein de ce ministère contribue également, dès sa première réunion ${ }^{65}$, à la formulation d'avis sur l'enseignement des gestes de premiers secours. La même année, le ministère de l'Intérieur institue une commission nationale de secourisme au sein de laquelle sont représentées les principales associations, afin d'élaborer le contenu du programme d'enseignement aux secouristes et moniteurs : à côté de la seule méthode manuelle de Nielsen, le bouche-à-bouche est la méthode « orale » retenue pour l'enseignement officiel des secouristes français. La Santé insiste sur la nécessité d'exclure $\mathrm{du}$ programme certaines techniques (inhalations de carbogène, utilisation des appareils de Cot et de Panis), considérées comme " périmées ${ }^{66}$ » et assimilables dans certains cas à des erreurs de traitement ${ }^{67}$, tandis qu'elles sont encore utilisées en 1960 dans les corps de sapeurs-pompiers ${ }^{68}$. Les débats sur la standardisation des méthodes peuvent donc se lire à travers les conflits institutionnels entre les ministères de la Santé et de l'Intérieur. 
Enseigner les techniques d'urgence : la standardisation comme enjeu pédagogique

Si bouche-à-bouche et massage cardiaque externe sont retenus par les différentes commissions chargées de les homologuer, c'est en raison de leur efficacité, mais aussi de la simplicité de leur exécution par un grand public préalablement averti. Les grandes associations de secourisme adoptent progressivement le bouche-à-bouche, mais l'expérience la plus remarquable revient sans doute à l'Ordre de Malte qui, dès l'été 1963, organise la campagne $\mathrm{du}$ « don du souffle ». Il s'agit d'inciter largement la population, dès son plus âge, à se familiariser avec cette technique de premiers secours, en organisant des démonstrations avec le mannequin Ressusci-Anne dans les lieux de villégiature ${ }^{69}$, puis dans des entreprises et sites publics les années suivantes. Engagée dans cette campagne, le Docteur Noviant réalise des films présentant le bouche-à-bouche, projetés au grand public dans les salles de cinéma ou utilisés lors de séances de formation de secouristes ${ }^{70}$. Elle insiste aussi sur la formation des personnels hospitaliers : il arrive trop souvent, en effet, que des patients meurent à l'hôpital, faute de premiers secours ${ }^{71}$. Il faut ici noter la volonté d'éducation sanitaire de jeunes médecins dont la spécialité médicale est en cours de consolidation, par l'intégration inédite de principes secouristes dans leur cursus. En prévoyant pour eux des stages dans les services de réanimation, les étudiants en médecine se voient pleinement intégrés à la volonté de diffusion des techniques de soins d'urgence.
Les manuels de premiers secours jouent alors un rôle essentiel dans le processus de vulgarisation des découvertes médicales validées par les experts ${ }^{72}$. Le bouche-à-bouche et le massage cardiaque externe font ainsi leur entrée - ou, plutôt, acquièrent une place de choix - dans cette littérature à destination des secouristes en formation ou déjà formés, mais aussi d'infirmiers et médecins soucieux d'avoir à portée de main un vade mecum à jour. Il s'agit de rendre compte, dans un langage simple et concis, des procédés de réanimation, au moyen si possible d'images et de schémas. Comme l'indique Christelle Rabier au sujet de la publication du geste chirurgical, ces illustrations à valeur pédagogique ont une forte dimension narrative, dans la mesure où elles cherchent à montrer « la dimension temporelle du geste ${ }^{73} "$, en le découpant, par exemple, en séquences qui correspondent chacune à une étape du processus à réaliser. Dans la première moitié des années 1960, plusieurs revues spécialisées en sécurité au travail deviennent également de véritables tribunes pour les médecins chargés d'enseigner les méthodes de secourisme et soucieux de faire connaître largement les innovations en matière de premiers secours. Chez EDF en particulier, le docteur François introduit de manière précoce l'enseignement du bouche-àbouche dans les écoles d'apprentissage et lors des sessions de formation permanente des agents. Le médecin utilise également la revue de l'entreprise afin de présenter la nouvelle méthode dans un long article ${ }^{74}$, tandis qu'à partir d'avril 1963, un encart « secourisme » explicite chaque mois un point de méthode aux lecteurs. 
Tandis que le bouche-à-bouche s'impose comme la méthode universelle de respiration artificielle, l'enseignement du massage cardiaque externe suscite un débat parmi les spécialistes, aux ÉtatsUnis comme en France (fig. 3). Dans la mesure où son succès dépend de la rapidité de sa mise en œuvre, certains estiment que le massage cardiaque devrait être appris par tout le monde, tandis que d'autres se focalisent sur ses dangers et pensent le réserver aux seuls initiés, capables de déceler avec certitude l'arrêt cardiaque par ses trois signes (apnée, absence de pouls, mydriase). La pratique du massage cardiaque est donc conditionnée par un diagnostic que seuls des initiés peuvent poser. Sur la proposition du service médical de la Protection civile, les sapeurs-pompiers secouristes d'une dizaine de départements français sont alors observés afin de déterminer si l'enseignement de la méthode peut être

généralisé aux trois cent mille secouristes du pays. La conclusion provisoire de l'enquête, en 1965, indique que seuls les secouristes spécialisés en " ranimation » peuvent pratiquer le massage cardiaque externe, les simples brevetés devant néanmoins connaître la méthode et les risques de son utilisation ${ }^{75}$. Le massage cardiaque demeure ainsi réservé aux experts jusqu'en 1978, tandis que le bouche-à-bouche est désormais présenté comme le geste universel du secours.

L'étude de la fabrication d'un consensus autour de la valeur du boucheà-bouche et le massage cardiaque externe suggère un déplacement d'un questionnement autour de l'efficacité du geste vers celui de sa normalisation, ou de son utilisation partagée par le monde secouriste. Leur standardisation est le fruit d'un processus, marqué par les conflits de légitimité des instances liées au

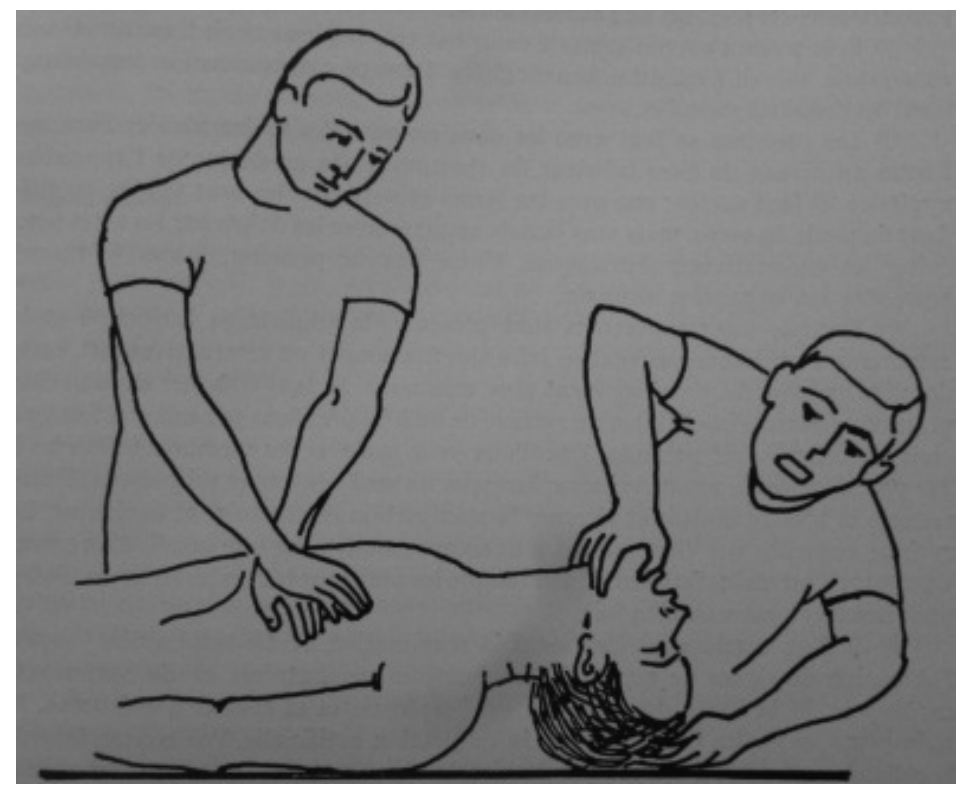

Figure 3. - Technique du massage cardiaque externe. Yvonne Noviant, « Principes et méthodes de ressuscitation pulmonaire et cardiaque », Analgésie, anesthésie, réanimation, vol. 19, 1962, p. 195. 
secourisme, de plus en plus nombreuses, et de personnalités médicales liées à des réseaux transnationaux. Acteurs privés ou étatiques, secouristes ou médicaux, trouvent un compromis autour de ces deux techniques, dont la maîtrise pose la question de la répartition des savoirs et des compétences. Le ministère de la Santé, dont la visibilité s'accroît à partir des années 1960, contribue à alimenter le débat en tentant d'intégrer les secouristes à une hiérarchie des personnels médicaux et paramédicaux. L'interaction entre ces institutions diverses - associations, ministères, académies - tend alors à placer le monde secouriste dans une économie de la santé dont ils constituent un pan bénévole.

Si la standardisation des gestes d'urgence entraîne une forme de dépendance des secouristes vis-à-vis du corps médical et des autorités sanitaires, elle révèle en même temps la valeur de ces acteurs spécifiques pour une collectivité soucieuse de la sauvegarde de la vie, en tant que détenteurs de techniques. Une étude sur l'utilisation de ces gestes par le corps médical dans les années 1970 suggère que l'éducation aux premiers soins est un chantier en cours : dans son enquête sur le rapport qu'entretiennent les médecins avec le secourisme, Philippe Duprat conclut que la moitié des praticiens ayant répondu ont une mauvaise connaissance de gestes d'urgence élémentaires qu'une partie de la population connaît - environ deux millions de secouristes non médecins ${ }^{76}$. Ainsi, au moment où se stabilisent ces savoirs, la frontière entre experts et profanes est plus que jamais remise en cause par l'existence d'associations secouristes et le zèle de sauveteurs, devenus techniciens de l'urgence vitale.

\section{Notes}

1. Roger Cooter, Bill Luckin (éd.), Accidents in history : injuries, fatalities, and social relations, Amsterdam and Atlanta, Editions Rodopi, 1997; Catherine DENYS, « La mort accidentelle à Lille et Douai au XVIII $^{\mathrm{e}}$ siècle : mesure du risque et apparition d'une politique de prévention ", Histoire urbaine, vol. 2, $\mathrm{n}^{\circ}$ 2, 2000, p. 95-112; Frédéric CAILle, La figure du sauveteur : naissance du citoyen secoureur en France (1780-1914), Rennes, Presses universitaires de Rennes, 2006.

2. Maurice LÉvy-Leboyer, Henri Morsel (dir.), Histoire générale de l'électricité en France, Paris, Association pour l'histoire de l'électricité en France, Fayard, 1994.

3. Philippe Leveau, « Le massage cardiaque : évolution des techniques ", Histoire des sciences médicales, 32, 2, 1998, p. 151-190.

4. Anne CARol, Les médecins et la mort: XIX ${ }^{e}-$ $\mathrm{XX}^{e}$ siècle, Paris, Aubier, 2004.

5. Victor RAymond et André Vallaud, L'oxyde de carbone et l'oxy-carbonisme, Paris, INS, 1949, cité in "Formation de moniteurs de respiration artificielle manuelle ", Protection, sécurité, hygiène dans l'atelier, $n^{\circ} 3$, mars 1964, p. 4.

6. Jean-Claude ChesnAIs, « La mortalité par accidents en France depuis 1826 », Population, n ${ }^{\circ} 6$, 1974, p. 1097-1136.

7. Jean-Claude ChesnAIs, Les morts violentes en France depuis 1826 : comparaisons internationales, Travaux et documents de l'Institut national d'études démographiques, Paris, Presses universitaires de France, 1976.

8. Roger Cooter, « Medicine and the goodness of war ", Canadian Bulletin of medical history, vol. 7, 1990, p. 147-159.

9. Guillaume LACHEnAL, Céline Lefìve et Vinh-Kim Nguyen (éd.), La médecine du tri : histoire, éthique, anthropologie, Paris, Presses universitaires de France, 2014.

10. Eliot Freidson, Professional dominance : the social structure of medical care, New York, Adline, 1970.

11. Décret n 64-830 du 5 août 1964.

12. Julia E. Szymczak et Charles L. Bosk, " Training for efficiency : work, time and systems-based practice in medical residency ", Journal of health and social behavior, vol. 53, $\mathrm{n}^{\circ} 3$, 2012, p. 344-358.

13. Stefan Timmermans et Marc Berg, "Standardization in action : achieving local universality through medical protocols ", Social studies of science, vol. 27, 2, 1997, p. 273-305.

14. Jacques Revel et Jean-Pierre Peter, « Le corps. L'homme malade et son histoire ", in 
Jacques Le Goff, Pierre Nora (dir.), Faire de l'histoire. Nouveaux problèmes, nouvelles approches, nouveaux objets, Paris, Gallimard, 1974, p. 848-873.

15. Les numéros de la revue Anesthésie, analgésie, réanimation, 1'une des deux revues françaises d'anesthésiologie, ont été consultés sur la période 1957-1973.

16. Jean-Pierre Le Crom, « La Croix-Rouge française pendant la Seconde Guerre mondiale ", Vingtième Siècle, Revue d'histoire, vol. 101, 1, 2008, p. 149-162.

17. AN (Pierrefitte), 19790386/1, chiffres établis à partir de leurs publications et des rapports de la Protection civile.

18. Pour le cas britannique, par exemple, Matthew Grant, After the bomb : civil defence and nuclear war in Britain, 1945-1968, Basingstoke, Royaume-Uni, 2010.

19. Le docteur Schafer est professeur de physiologie à la faculté d'Edimbourg. Voir Charles COT, École pratique du secouriste spécialisé dans les soins aux asphyxiés du temps de paix et aux gazés du temps de guerre, Paris, Le François, 1935.

20. Blandine BRIL, «L'apprentissage de gestes techniques : ordre de contraintes et variations culturelles ", "Le geste technique. Réflexions méthodologiques et anthropologiques ", Technologies, idéologies, pratiques, vol. 14, n², 2002, p. 113-149.

21. Jean-Pierre Williot insiste sur la baisse du prix du gaz et sur le développement des applications domestiques du gaz (cuisine, chauffage) ; Jean-Pierre Williot, Naissance d'un service public : le gaz à Paris, Paris, Éditions Rive droite, 1999; id., «La respiration des gazomètres ou la consommation de gaz manufacturé à Paris de 1860 à 1960 », Flux, 51, 2003, p. 83-88.

22. Charles Сот, Les asphyxies accidentelles : submersion, électrocution, intoxication oxycarbonique, étude clinique, thérapeutique et préventive, Paris, Maloine, 1931.

23. Docteur Lartigue, « Insuffisance des statistiques relatives aux accidents syncopoasphyxiques. Modèle de fiche statistique ", Le Devoir, 189, mars-avril 1956, p. 295-297.

24. Pierre MARION et Raymond Deleuze, «Choix d'un procédé de respiration artificielle par manœuvres externes », Anesthésie, analgésie, réanimation, vol. 14, 1957, p. 268.

25. Michel SÉclin, Contribution à l'étude des méthodes manuelles de respiration artificielle applicables au traitement d'urgence, thèse de médecine, Paris, 1951, qui propose une synthèse en français des études comparées des différentes méthodes et de leur efficacité.

26. Vœu adopté à l'unanimité par l'Académie de médecine, le 6 juillet 1954 .
27. Voir le numéro " Fitting for Health ", Technology and Culture, vol. 54, 3, 2013.

28. D'après les chiffres indiqués dans les Statistique des incendies et des sauvetages pour lesquels le régiment de sapeurs-pompiers de Paris a été appelé, les sapeurs-pompiers seraient intervenus à 400 reprises dans les hôpitaux parisiens entre 1932 et 1941 .

29. On peut également retracer leur histoire à travers les brevets publiés.

30. «Appareil pour respiration artificielle de M. Germain Panis », présenté par le professeur Léon Bernard, Bulletin de l'Académie nationale de médecine, vol. 89, 3, 1923, p. 472.

31. Bruno J. G. De TuRCK, « The resuscitation great. Charles Hederer and his pulmoventilateur ", Resuscitation, 79, 2008, p. 7-10.

32. Anson RABINBACH, Le moteur humain : l'énergie, la fatigue et les origines de la modernité, Paris, La Fabrique, 2004.

33. Christine Bergé, " "Monsieur, je vous

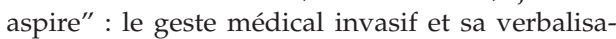
tion ", in Anne-Claude Ambroise-Rendu, Fabrice D'Almeida, Nicole Edelman, Annette BecKer, et Christian Delporte, Des gestes en histoire : formes et significations des gestualités médicale, guerrière et politique, Paris, Seli Arslan, 2006, p. 68-84.

34. Charles Сот, École pratique du secouriste spécialisé dans les soins aux asphyxiés du temps de paix et aux gazés du temps de guerre, Paris, Le François, 1935.

35. Isabelle MicLOT, « Émotions nucléaires. La population française face à la menace de guerre nucléaire 1950-1960 », in Philippe Buton, Olivier Büttner, Michel Hastings (dir.), La Guerre froide vue d'en bas, Paris, CNRS Éditions, 2014, p. 289307.

36. Sur l'histoire de la réanimation, voir le témoignage de Maurice RAPIN, Retrouver la vie : histoire, pouvoir et limites de la réanimation, Paris, Robert Laffont, 1980; Éric BRET, « Plasticité des spécialités. De la phtisiologie à la pneumologie et naissance de la réanimation », Actes de la recherche en sciences sociales, 156-157, 2005, p. 88-97.

37. Vincent Mouret, Histoire de la poliomyélite, thèse de médecine, université de Limoges, 1998.

38. Plusieurs témoignages rendent compte de cette expérience, par exemple Rosanna BENZI, La cuirasse : trente ans dans un poumon d'acier, traduit de l'italien par Irène Limon, Paris, Presses de la Cité, 1992.

39. Un phénomène similaire est à l'œuvre dans le domaine de la défibrillation cardiaque, avec la réduction de la taille des appareils; Michel Chauvin, Le geste qui sauve, l'étonnante histoire du défibrillateur cardiaque externe, Paris, L'Harmattan, 2016. 
40. Maurice CARA, Michel Poisvert et al., « Le transport d'urgence des asphyxiques par les médecins du laboratoire expérimental de physique de l'AP à Paris ", Anesthésie, analgésie, réanimation, vol. 14, 1957, p. 942-956.

41. Jean Natali, « Éloge de Maurice Cara (1917-2009) », Bulletin de l'Académie nationale de médecine, vol. 195, 7, 2011, p. 1455.

42. Joseph Rechman, «William Buchan (17291805). Le bouche à bouche et le massage cardiaque externe ", Histoire des sciences médicales, 13, 3, 1979, p. 291.

43 P. J. BAsKetT, " The resuscitation greats. Peter J. Safar, the early years 1924-1961, the birth of CPR », Resuscitation, 50, 2001, p. 17-22.

44. Stefan Timmermans, Sudden death and the myth of CPR, Philadelphia, Temple University Press, 2010.

45. Parmi les nombreux articles publiés à partir de 1958 sur cette méthode, on retiendra le premier : P. SAFAR, L. A. Escarraga, J. O. ElAm, " Comparison of mouth-to-Mouth and mouthto-airway methods of artificial respiration with chest-pressure arm-lift methods ", New England Journal of medicine, 258, 1958, p. 671-677.

46. Yonne Noviant, "Souvenirs entremêlés ", in Ernest KerN, Jean LAssner, Guy Vourc'H (dir.), Regard sur l'anesthésie d'hier, Paris, Glyphe \& Biotem, 2003, p. 207-211. Sur l'histoire de la discipline en France, Marie-Thérèse Cousin, L'anesthésie-réanimation en France : des origines à 1965, Paris, L'Harmattan, 2005.

47. Yvonne Noviant, «Principes et méthodes de ressuscitation pulmonaire et cardiaque ", Analgésie, anesthésie, réanimation, vol. 19, 1962, p. 183-199. C'est d'ailleurs pour ses travaux sur la respiration artificielle et notamment sur le bouche-à-bouche que l'Académie de médecine lui décerne le prix Crapet en 1964.

48. AN, 19790386/9, procès-verbal de la réunion tenue le 6 février 1959 au ministère de la Santé publique et de la population relative aux premiers secours aux électrocutés.

49. Robert P. SANDS, Douglas R. BACON, «An inventive mind : the career of James O. Elam, M. D. (1918-1995) », Anesthesiology, 88, 4, 1998, p. $1107-1112$.

50. Boehm est présenté par les historiens de la médecine comme étant le premier à décrire le massage cardiaque externe en 1878 : il obtient la réanimation de chiens et de chats par la ventilation artificielle associée à des pressions rythmiques sur le thorax. König applique cette technique à l'homme en 1883, connue plus tard sous le nom de Mass-König : elle repose sur l'idée que la compression du cœur entre le sternum et le rachis permet de générer un mouvement semblable à la systole. Cette «théorie de la pompe cardiaque ", remise en cause plus tard dans les années 1970, est cependant celle qui sous-tend la reconnaissance du massage cardiaque externe comme méthode efficace de réanimation cardiaque; Ph. LEveAu, "Le massage cardiaque : évolution des techniques ", op. cit.

51 W. B. Kouwenhoven, J. R. Jude et G. G. KNICKerbocker, « Closed-chest cardiac massage », JAMA, 173, 1960, p. 1064-1067.

52. Yann FAURE, « L'anesthésie française entre reconnaissance et stigmates ", Actes de la recherche en sciences sociales, 156-157, 2005, p. 98-114.

53. Marcel Mauss, "Techniques du corps ", Journal de psychologie, vol. 32, 15 mars-15 avril 1936.

54. Pierre-André Delaby, « À propos des appareillages de réanimation ", Histoire des sciences médicales, 13, 3, 1979, p. 299-310.

55. Alain CoRbIN, « Histoire et anthropologie sensorielle », Anthropologie et sociétés, vol. 14, 2, 1990, p. 13-24.

56. AN, 19790386/9, note du médecin-général Genaud au Service national de la protection civile, 8 septembre 1958 .

57. Didier FAssin et Patrice Bourdelais (éd.), Les constructions de l'intolérable : études d'anthropologie et d'histoire sur les frontières de l'espace moral, Paris, La Découverte, 2005.

58. Arne RubEN, « Les principes et les méthodes d'insufflation avec l'appareil d'Ambu et par la méthode bouche-à-bouche ", Anesthésie, analgésie, réanimation, vol. 17, 1960, p. 70-71.

59. Gilbert Simondon, Du mode d'existence des objets techniques, Paris, Aubier, 1958.

60. Nina TJomsland, Peter BAsKett, « The resuscitation greats. Åsmund S. Lærdal ", Resuscitation, 53, 2002, p. 115-119.

61. David MendonçA, Gary Webb, Carter ButTs, " L'improvisation dans les interventions d'urgence : les relations entre cognition, comportement et interactions sociales ", Tracés. Revue de sciences humaines, 18, 2010, mis en ligne le 01 mai 2012, consulté le 22 juillet 2016.

62. Roger CORNU, "Voir et savoir », in Denis Chevallier (dir.), Savoir faire et pouvoir transmettre : transmission et apprentissage des savoir-faire et des techniques [actes des rencontres de Royaumont, 15-17 janvier 1990], Paris, Éd. de la Maison des sciences de l'homme (Collection Ethnologie de la France), 1991, p. 83-100.

63. Luc Berlivet, " Médicalisation ", Genèses, 82,2011, p. 2-6.

64. 8634 tués et 146083 blessés sur les routes en 1953, respectivement 12081 et 238900 blessés 
en 1963 ; Jacques VAllin, Jean-Claude ChEsnAIs, « Les accidents de la route en France. Mortalité et morbidité depuis 1953 », Population, n³, 1975, p. 443-478.

65. AN, 20080612/1, compte rendu de la réunion de la commission de réanimation, 4 mai 1964.

66. AN, 19790386/5, lettre du ministre de la Santé au ministre de l'Intérieur, 2 décembre 1964.

67. Charles L. Bosk, Forgive and remember : managing medical failure, $2^{\mathrm{e}}$ éd. Chicago, University of Chicago Press, 2003.

68. Archives municipales de Reims, 39 W 41. En témoigne la liste du matériel précisée sur la demande d'agrément que formule le corps des sapeurs-pompiers de la ville de Reims au Service national de la protection civile, 2 mars 1960.

69. "Campagne du don du souffle », Bulletin de l'Ordre de Malte, 1, janvier-juillet 1963, p. 6-7.

70. Entretien avec le professeur Pierre Jolis, le 11 juillet 2016; entretien téléphonique avec Marc Wapler, fils du docteur Noviant, le 22 juillet 2016. Il rappelle que sa mère l'a sollicité pour participer au tournage d'un des films, dans le rôle d'un enfant inanimé.

71. Yvonne Noviant, «Enseignement hospitalier de la ressuscitation de première urgence ", Anesthésie, analgésie, réanimation, vol. 19, 1962, p. 259-265.
72. En dehors des manuels publiés par la Protection civile, il faut noter le premier manuel rédigé par des anesthésistes en 1963 : Maurice CAra et Michel Poisvert, Premiers secours dans les détresses respiratoires : traitements d'urgence des formes aiguës au cours des maladies, des intoxications et des accidents, Paris, Masson, 1963.

73. Christelle Rabier, « Publier le geste chirurgical : la lithotomie en France et au Royaume-Uni (1720-1820) », in A.-Cl. Ambroise-Rendu et al., Des gestes en histoire : formes et significations des gestualités médicale, guerrière et politique, op. cit., p. 33.

74. Docteur FrançOIs, "Réanimation. Les méthodes modernes - Possibilités et limites d'application au cas de l'accidenté électrique ", Vigilance, 23, octobre 1963, p. 25-32, repris dans la revue de l'Association des industriels de France contre les accidents du travail, Protection, sécurité, hygiène du travail, 5, mai 1964, p. 63-74.

75. MM. Genaud, Legendre et Saury, "Massage cardiaque externe et noyade ", Bulletin de l'Académie nationale de médecine, vol. 149, 5-6, 1965, p. 106.

76. Ce résultat est obtenu à partir de 317 réponses sur un échantillon de 1000 médecins dont les noms ont été choisis au hasard dans un annuaire médical; Philippe Duprat, Le médecin praticien et les gestes de premiers secours, thèse de médecine, Broussais-Hôtel-Dieu, 1976. 